[Article]

\title{
分子转动和激光脉冲对多光子激发控制的影响
}

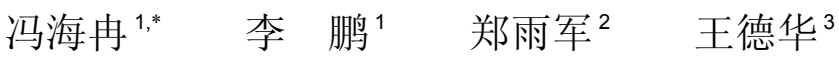 \\ ('济宁学院物理与信息工程系, 山东曲阜 273155; 2 2 山东大学物理学院, 济南 250100; \\ 3鲁东大学物理与光电工程学院, 山东烟台 264025)
}

\begin{abstract}
摘要：用解析代数方法研究了分子转动和激光脉冲对双原子分子多光子激发控制的影响并推导得到不同转 动通道下的分子振动激发几率的解析表达式. 为了考察转动能级和考虑分子转动后与激光场夹角的变化对分 子多光子振动激发和振动激发控制的影响, 我们计算并比较了分子纯振动和加入分子转动两种情况, 并分别给 出了分子与极化激光场在不同取向角下三光子选择激发的图像. 研究发现分子的转动能级对多光子非共振激 发有修正作用, 但是分子转动会降低多光子激发的选择性, 而选择合适的激光脉冲形状有利于目标多光子激发 控制的实现. 文中还进一步讨论了激光脉冲初相位对分子多光子激发控制的影响, 发现脉冲初相位对多光子激 发过程有明显的调制作用.
\end{abstract}

关键词: 多光子; 分子; 激发; 控制; 李代数

中图分类号: 0644.1; O561.4

\section{Influences of Molecular Rotations and Laser Pulses on Controlling Multiphoton Excitation}

\author{
FENG Hai-Ran ${ }^{1, *} \quad$ LI Peng ${ }^{1} \quad$ ZHENG Yu-Jun ${ }^{2} \quad$ WANG De-Hua ${ }^{3}$ \\ ('Department of Physics and Information Engineering, Jining University, Qufu 273155, Shandong Province, P. R. China; \\ ${ }^{2}$ School of Physics, Shandong University, Jinan 250100, P. R. China; ${ }^{3}$ School of Physics and Optoelectronic Engineering, \\ Ludong University, Yantai 264025, Shandong Province, P. R. China)
}

\begin{abstract}
The influence of molecular rotation, laser pulse shape and initial phase on controlling the infrared multiphoton excitation of diatomic molecules has been studied using an analytical algebraic approach, which involved the derivation of analytic transition probabilities with various rotational channels. To observe the correctional functions of the rotational energy and the relationship between the molecular orientation and the polarized direction of the laser field in terms of their impact on controlling multiphoton excitation, we calculated the probabilities in the purely vibrational and ro-vibrational cases. The maximum transition probabilities were determined as a function of the time and molecular orientation angle in both cases for comparison, which allowed for the target multiphoton excitations to be achieved. However, oscillations appeared in the population of the ro-vibrational case which denoted rotational interference can decrease the selectivity of the molecular vibrational excitation. Furthermore, the rotational energy had a corrected action on multiphoton non-resonant excitation and the power of actions was dependent on the molecular anharmonicity. We have also provided a discussion of the influences of laser pulse shape and initial phase. We found that the use of an appropriate laser pluse shape afforded the target multiphoton excitation event, and that the initial phase of the chirped laser pulse had an obvious modulatory function on the multiphoton processes.
\end{abstract}

Key Words: Multiphoton; Molecule; Excitation; Control; Lie-algebra

\footnotetext{
Received: December 30, 2013; Revised: March 25, 2014; Published on Web: March 26, 2014.

"Corresponding author. Email: hrfeng_jnxy@163.com; Tel: +86-537-3196107.

The project was supported by the National Natural Science Foundation of China $(11147019,91021009,11374133)$.

国家自然科学基金(11147019, 91021009, 11374133)资助项目

(c) Editorial office of Acta Physico-Chimica Sinica
} 


\section{Introduction}

Molecular multiphoton processes have been researched hotspot for more than 20 years. Considerable interest has been focused on the influence of molecular-orientation on multiphoton processes. ${ }^{1-8}$ Considering molecular rotational motion, the orientation of molecules relative to the polarization of a laser field is an important factor for the control of multiphoton excitations. However, most theoretical works have examined the problem by solving the time-dependent Schrödinger equation. Few analytical studies have been conducted on the influence of molecular rotation on the control of multiphoton processes. For the past years, applications of the dynamic Lie-algebraic approach have steadily advanced..$^{9-15}$ Algebraic methods have been extensively used to investigate problems in nuclear physics, molecular physics, and quantum optics, etc. In the algebraic framework, the Hamiltonian system is given using a set of dynamic algebra, and the time-evolution operator of the quantum system can be directly obtained only if operators in the Hamiltonian close under communication with every element of the dynamic algebra. Computational time is saved by avoiding the solution of the time-dependent Schrödinger equation, and analytical expression of the vibrational transition probability can be achieved using the algebraic approach. We have successfully studied the control of vibrational excitation for small molecules using the Lie-algebraic approach. ${ }^{16,17}$ We have also discussed long-time average absorbed energy spectra and the average number of photons absorbed by the molecule in a normal sinusoidal laser field when rotational motions are considered. ${ }^{18}$ Given that the algebraic model can be expanded to discuss the influence of molecular rotation, we can also discuss the influence of rotation on controlling infrared multiphoton excitation. Control of multiphoton vibrational excitations can enable selective vibrational transition and dissociation on demand, which helps regulate the chemical reaction and the preparation of quantum bits.

Studying the influence of molecular rotations on controlling infrared multiphoton excitation can help elucidate not only the interference effect of molecular alignment and orientation but also the impact of rotational energies, hence, the two aspects are emphases of our research and the rotational excitations are not considered here. In this study, the influence of rotation on controlling infrared multiphoton excitation in diatomic molecules is studied by the analytical algebraic approach. Transition probability with various rotational channels is analytically given using the method. To manifest the influence of rotations, we first study multiphoton resonant excitation in a normal sinusoidal laser field, considering both pure vibrational and ro-vibrational modes. We then discuss the selective multiphoton excitation of two modes in a chirped and shaped laser field. The parameters of laser pulses are also vital factors affecting quantum control. Accordingly, the influences of laser shape and initial laser phase are also studied.

\section{Theoretical framework}

The Hamiltonian of the system is

$\mathbf{H}=\mathbf{H}_{\mathrm{m}}+\mathbf{H}_{\mathrm{i}}$

where $\mathbf{H}_{\mathrm{m}}$ represents the Hamiltonian of free vibrational-rotational diatomic molecule and $\mathbf{H}_{\mathrm{i}}$ is its interaction Hamiltonian with a laser field.

According to the quadratic anharmonic Lie-algebraic mod$\mathrm{el},{ }^{19-22}$

$$
\begin{aligned}
& \mathbf{H}_{\mathrm{m}}=\hbar \omega_{0}\left(\mathbf{A}_{+} \mathbf{A}_{-}+\frac{\mathbf{I}_{0}}{2}\right)+\frac{l(l+1) \hbar^{2}}{2 m r^{2}} \\
& \mathbf{H}_{\mathrm{i}}=\boldsymbol{\mu} \times \varepsilon(t)
\end{aligned}
$$

where $\hbar$ is Planck's constants divided by $2 \pi, m$ is the reduced mass of molecules, $r$ is the distance between two nuclei, $r_{0}$ is the distance at equilibrium, $l$ is the angular moment quantum number, $\varepsilon(t)$ is the polarized laser field, and $\boldsymbol{\mu}$ is the molecular dipole moment function.

Both rotational term in the molecular Hamiltonian and the molecular dipole moment can be expanded in a series at equilibrium

$$
\frac{1}{r^{2}} \cong \frac{1}{r_{0}^{2}}-\frac{2}{r_{0}^{3}}\left(r-r_{0}\right)=\frac{1}{r_{0}^{2}}-\frac{2}{r_{0}^{3}} \mathbf{x}
$$

$$
\mu(\mathbf{x})=\mu \mathbf{x}
$$

where $^{19}$

$$
\mathbf{x}=\sqrt{\frac{\hbar \omega_{0}}{D}} \frac{1}{2 \alpha}\left(\mathbf{A}_{+}+\mathbf{A}_{-}\right)
$$

where $r_{0}$ is the distance at equilibrium, $D$ is the dissociation energy, and $\alpha$ is the Morse parameter.

Thus the Hamiltonian operator of the system can be written as

$$
\mathbf{H}=\hbar \omega_{0}\left(\mathbf{A}_{+} \mathbf{A}_{-}+\frac{\mathbf{I}_{0}}{2}\right)+d_{l}\left(\mathbf{A}_{+}+\mathbf{A}_{-}\right)+l_{0}
$$

where

$$
\begin{aligned}
& d_{l}(t)=\sqrt{\frac{\hbar \omega_{0}}{D}} \frac{\Omega(t)}{2 \alpha}-\sqrt{\frac{\hbar \omega_{0}}{D}} \frac{l(l+1) \hbar^{2}}{2 m r_{0}^{3} \alpha} \\
& \Omega(t)=-\mu \varepsilon(t) \cos \theta \\
& l_{0}=\frac{l(l+1) \hbar^{2}}{2 m r_{0}^{2}}
\end{aligned}
$$

where $\theta$ is the angle between the molecular orientation and the axis of the polarized field.

Given that we choose

$$
\begin{aligned}
& \mathbf{H}_{0}=\hbar \omega_{0}\left(\mathbf{A}_{+} \mathbf{A}_{-}+\frac{\mathbf{I}_{0}}{2}\right) \\
& \mathbf{V}=d_{l}\left(\mathbf{A}_{+}+\mathbf{A}_{-}\right)+l_{0}
\end{aligned}
$$

the Hamiltonian system (Eq.(1)) in the interaction picture reads as

$$
\begin{aligned}
\mathbf{H}_{I}(t) & =\mathrm{e}^{\mathrm{i} \mathbf{H}_{0} t / \hbar} \mathbf{V} \mathrm{e}^{-i \mathbf{H}_{0} t / \hbar} \\
& =\mathrm{e}^{\mathrm{i} \omega_{0} \chi_{0} \mathbf{A}_{A_{+}} \mathbf{A}_{-}} \mathrm{e}^{\mathrm{i} \omega_{0} \mathbf{I}_{0} / 2}\left[d_{l}\left(\mathbf{A}_{+}+\mathbf{A}_{-}\right)+l_{0}\right] \mathrm{e}^{-i \omega_{0} \mathbf{I}_{0} / 2} \mathrm{e}^{-i \omega_{0} \chi_{0} t \mathbf{A}_{+} \mathbf{A}} \\
& =d_{l} \mathrm{e}^{\mathrm{i} \omega_{0} \chi_{0} t} \mathrm{e}^{\mathrm{i} \omega_{0} \mathbf{I}_{0} t} \mathbf{A}_{+}+d_{l} \mathrm{e}^{\mathrm{i} \omega_{0} \chi_{0} t} \mathrm{e}^{-i \omega_{0} \mathbf{I}_{0} t} \mathbf{A}_{-}+l_{0} \mathbf{A}_{0} \\
& =\gamma_{+} \mathbf{A}_{+}+\gamma_{-} \mathbf{A}_{-}+l_{0} \mathbf{A}_{0}
\end{aligned}
$$

where $\chi_{0}$ is the anharmonicity parameter, $\mathbf{A}_{0}$ is the identity operator and $\mathbf{I}_{0}, \mathbf{A}_{-}, \mathbf{A}_{+}$have communication relations

$$
\left[\mathbf{A}_{-}, \mathbf{A}_{+}\right]=I_{0},\left[\mathbf{A}_{ \pm}, \mathbf{I}_{0}\right]= \pm 2 \chi_{0} \mathbf{A}_{\text {干 }}
$$


dimensional Lie-algebra, the time evolution operator can be represented $\operatorname{as}^{23-25}$

$$
\mathbf{U}_{I}=\mathrm{e}^{-\frac{i}{\hbar} \mu_{0} \mathbf{A}_{0}} \mathrm{e}^{-\frac{i}{\hbar} \mu_{1} \mathbf{I}_{0}} \mathrm{e}^{-\frac{i}{\hbar} \mu_{2} \mathbf{A}_{+}} \mathrm{e}^{-\frac{i}{\hbar} \mu_{3} \mathbf{A}_{-}}
$$

The time-evolution operator $\mathbf{U}_{I}$ satisfies in the interaction picture

$$
\mathrm{i} \hbar \frac{\partial \mathbf{U}_{I}}{\partial t}=\mathbf{H}_{I} \mathbf{U}_{I}
$$

The set of differential equations can then be given by substituting Eq.(10) into Eq.(11)

$$
\begin{aligned}
& \dot{\mu}_{1}=-\frac{\mathrm{i}}{\hbar} \mu_{2} \gamma_{-} \mathrm{e}^{\frac{\mathrm{i}}{\hbar} 2 \chi_{0} \mu_{1}} \\
& \dot{\mu}_{2}=\gamma_{+} \mathrm{e}^{-\frac{\mathrm{i}}{\hbar} 2 \chi_{0} \mu_{1}}-\frac{x_{0}}{\hbar^{2}} \mu_{2}^{2} \gamma_{-} \mathrm{e}^{\frac{\mathrm{i}}{\hbar} 2 \chi_{0} \mu_{1}} \\
& \dot{\mu}_{3}=\gamma_{-} \mathrm{e}^{\frac{\mathrm{i}}{\hbar} 2 \gamma_{0} \mu_{1}} \\
& \dot{\mu}_{0}=l_{0}
\end{aligned}
$$

with the initial conditions

$$
\mu_{r}(t=0)=0 \quad(r=0,1,2,3)
$$

The time- dependent population probability from the initial ro-vibrational state $\mid v_{i}, l>$ to the target ro-vibrational state $\mid v_{f}, l>$ is

$$
\begin{aligned}
& P_{i f}^{l}(t)=\left|\left\langle v_{f}, l\left|\mathbf{U}_{I}(t)\right| v_{i}, l\right\rangle\right|^{2} \\
& P_{i f}^{l}=|\lambda|^{2} \delta_{v f, v i-j+k}
\end{aligned}
$$

where

$$
\begin{aligned}
\lambda= & \exp \left\{-\frac{\mathrm{i}}{\hbar} \mu_{1}\left[1-2 \chi_{0}\left(v_{i}-j+k\right)\right]-\frac{\mathrm{i}}{\hbar} \mu_{0}\right\} . \\
& \sum_{k=0}^{\infty} \frac{1}{k !}\left(-\frac{\mathrm{i}}{\hbar} \mu_{2}\right)^{k} \sqrt{\prod_{k^{\prime}=0}^{n}\left[1-\chi_{0}\left(v_{i}-j^{\prime}+k^{\prime}-1\right)\right]\left(v_{i}-j^{\prime}+k^{\prime}\right)} . \\
& \sum_{j=0}^{\infty} \frac{1}{j !}\left(-\frac{\mathrm{i}}{\hbar} \mu_{3}\right)^{j} \sqrt{\prod_{j^{\prime}=0}^{m}\left[1-\chi_{0}\left(v_{i}-j^{\prime}\right)\left(v_{i}-j^{\prime}+1\right)\right]}
\end{aligned}
$$

The analytical expression of transition probability in different ro-vibrational channels is obtained, and many concrete examples can be studied using this expression.

The corresponding long-time average probability is defined as

$$
\bar{P}_{i f}^{l}=\lim _{T \rightarrow \infty}\left\{\frac{1}{T} \int_{0}^{T} P_{i f}^{l}(t)\right\}
$$

\section{Results and discussion}

Here, we take $\mathrm{OH}$ and $\mathrm{OD}$ molecules as examples. All calculations are carried out using atomic units (a.u.). The parameters are taken from references, ${ }^{26-30}$ namely, $\omega_{0}=0.01664$ a.u., $\chi_{0}=$ 0.02323 a.u., $D=0.1614$ a.u., $\alpha=1.156$ a.u., $B_{\mathrm{e}}=0.8598 \times 10^{-5}$ a.u., $\alpha_{\mathrm{e}}=0.3253 \times 10^{-5}$ a.u., $D_{\mathrm{e}}=0.8748 \times 10^{-5}$ a.u. for $\mathrm{OH}$ and $\omega_{0}=$ 0.0122 a.u., $\chi_{0}=0.01645$ a.u., $D=0.1636$ a.u., $\alpha=1.142$ a.u., $B_{\mathrm{e}}=$ $0.4556 \times 10^{-5}$ a.u., $\alpha_{\mathrm{e}}=0.1321 \times 10^{-5}$ a.u., $D_{\mathrm{e}}=0.8748 \times 10^{-5}$ a.u. for OD. $\omega_{0}$ is the angular frequency of a anharmonic oscillator, $\chi_{0}$ is the corresponding anharmonicity parameter, $D$ is the dissociation energy, $\alpha$ is the Morse parameter, $B_{\mathrm{e}}, \alpha_{\mathrm{e}}$, and $D_{\mathrm{e}}$ are the corresponding rotational constants. First, we discuss molecular multiphoton excitations and provide a concrete comparison between purely vibrational and the rovibrational cases in the first subsection. We then study the influence of rotations and laser phases in controlling infrared multiphoton excitation. The dif- ferences in the optimum laser parameters are also given in the second subsection.

\subsection{Vibrational and ro-vibrational multiphoton excitations}

This subsection demonstrates the influence of rotation on molecular multiphoton excitations. To study the influence of rotation, we calculate the probabilities in the purely vibrational case (molecular orientation is aligned with the field) and in the ro-vibrational case $(l=1)$. In these cases, the laser field is $\varepsilon(t)=$ $\varepsilon_{0} \sin \left(\omega_{L} t\right)$. The laser intensity $\varepsilon_{0}$ is chosen as 0.0015 a.u., and the angle $\theta$ is averaged over $-\pi / 2-\pi / 2$ in the calculation. The initial state of the molecules is set at the ground state at $t=0$. Fig.1 and Fig.2 depict the long-time average probabilities of $\mathrm{OH}$ and $\mathrm{OD}$ from the ground state to the first, second, and third excited states using Eq.(17). According to the definition of multiphoton resonance transition, ${ }^{31}$

$$
\omega_{r} \cong \omega_{n}=\frac{\left(E_{f}-E_{0}\right)}{n \hbar}
$$

where $\omega_{r}$ is the resonant transition frequency, $\omega_{n}$ is the n-photon transition frequency, $E_{f}-E_{0}$ is the energy gap between the ground state and the $f$ th excited state, these resonant transitions correspond to one, two, and three- photon transitions, respec-

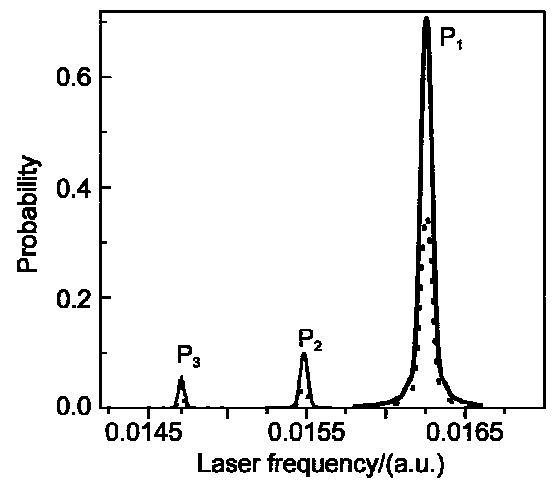

Fig.1 Long-time average probabilities from the ground to the first, second, and third states of $\mathrm{OH}$ as a function of laser frequency The solid curve denotes probabilities in purely vibrational calculation. The dot curve denotes probabilities in the ro-vibrational calculation.

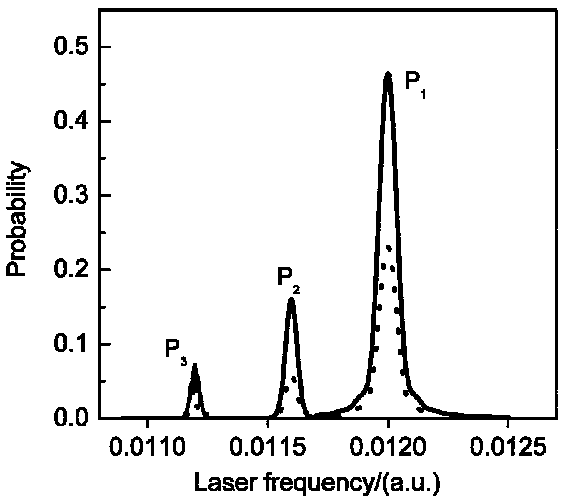

Fig.2 Long-time average probabilities from the ground to the first, second, and third states of $O D$ as a function of laser frequency The solid curve denotes probabilities in the purely vibrational calculation. The dot curve denotes probabilities in the ro-vibrational calculation. 
tively. $E_{f}$ is the energy eigenvalue that can be written as $\mathrm{s}^{30,32}$

$$
\begin{gathered}
E_{f}(v, l)=\hbar \omega_{0}\left[\left(v+\frac{1}{2}\right)-x_{0}\left(v+\frac{1}{2}\right)^{2}\right]+B_{\mathrm{e}} l(l+1)- \\
\alpha_{\mathrm{e}} l(l+1)\left(v+\frac{1}{2}\right)-\bar{D}_{\mathrm{e}}[l(l+1)]^{2}
\end{gathered}
$$

where the first two terms denote the anharmonic oscillator energy and the rigid rotor energy, and the last two terms represent vibration-rotation interaction and centrifugal distortion, respectively. Fig.1 and Fig.2 show that the average probabilities in the ro-vibrational case are much smaller than those in the purely vibrational case. We also calculate the transition probability in various rotational $l$-channels and find minimal changes in probability values. These findings indicate that the molecule experiences different orientations in the polarized laser field in the ro-vibrational case. Thus, the effective interaction strength in the ro-vibrational case is lower than that in the purely vibrational case. Thus, the molecular orientation in the laser field is very important to the ro-vibrational transition. This result coincides with previous ones. ${ }^{33,34}$

Few changes are observed in the values of the resonant frequencies for the two cases, but differences are still found upon more accurate calculations. The resonant frequency of the three-photon excitation in the ro-vibrational case changes from 0.014707 a.u. (in the purely vibrational case) to 0.0147069 a.u. for $\mathrm{OH}$ and 0.011197 a.u. (in the purely vibrational case) to
0.0111967 a.u. for OD. The shifting value of the resonant frequency is about $\omega_{L} \approx 0.1 \mathrm{~cm}^{-1}$, which may be due to correctional functions of the rotational energy for molecular vibrational anharmonicity. However, the rotational energy is still lower than the vibrational energy.

Fig. 3 and Fig. 4 show the time-dependent transition probabilities in the purely vibrational calculation $(l=0)$ and in the ro-vibrational calculation for $\mathrm{OH}$ and $\mathrm{OD}$ molecules $(l=1)$. The resonant probabilities are clearly found to have periodic behaviors. The resonant transition periods for the two cases are summarized in Table 1. For single-photon resonant transition, the corresponding period minimally changes in the two cases. However, the two and three-photon resonances are definitely long-time phenomena, consistent with the report of reference. ${ }^{35}$ Moreover, the periods of ro-vibrational transitions become longer than those of vibrational transitions in the multiphoton resonances because the rotational energy has the corrected action on molecular anharmonicity. Non-resonant multiphoton transitions also appear earlier in the purely vibrational case than in the ro-vibrational case. Compared with $\mathrm{OH}$ molecule, the period of multiphoton transition for the OD molecule becomes longer in the ro-vibrational case. In other words, the resonant periods have larger changes in the OD molecule than in the $\mathrm{OH}$ molecule when the rotational factor is considered. The reason
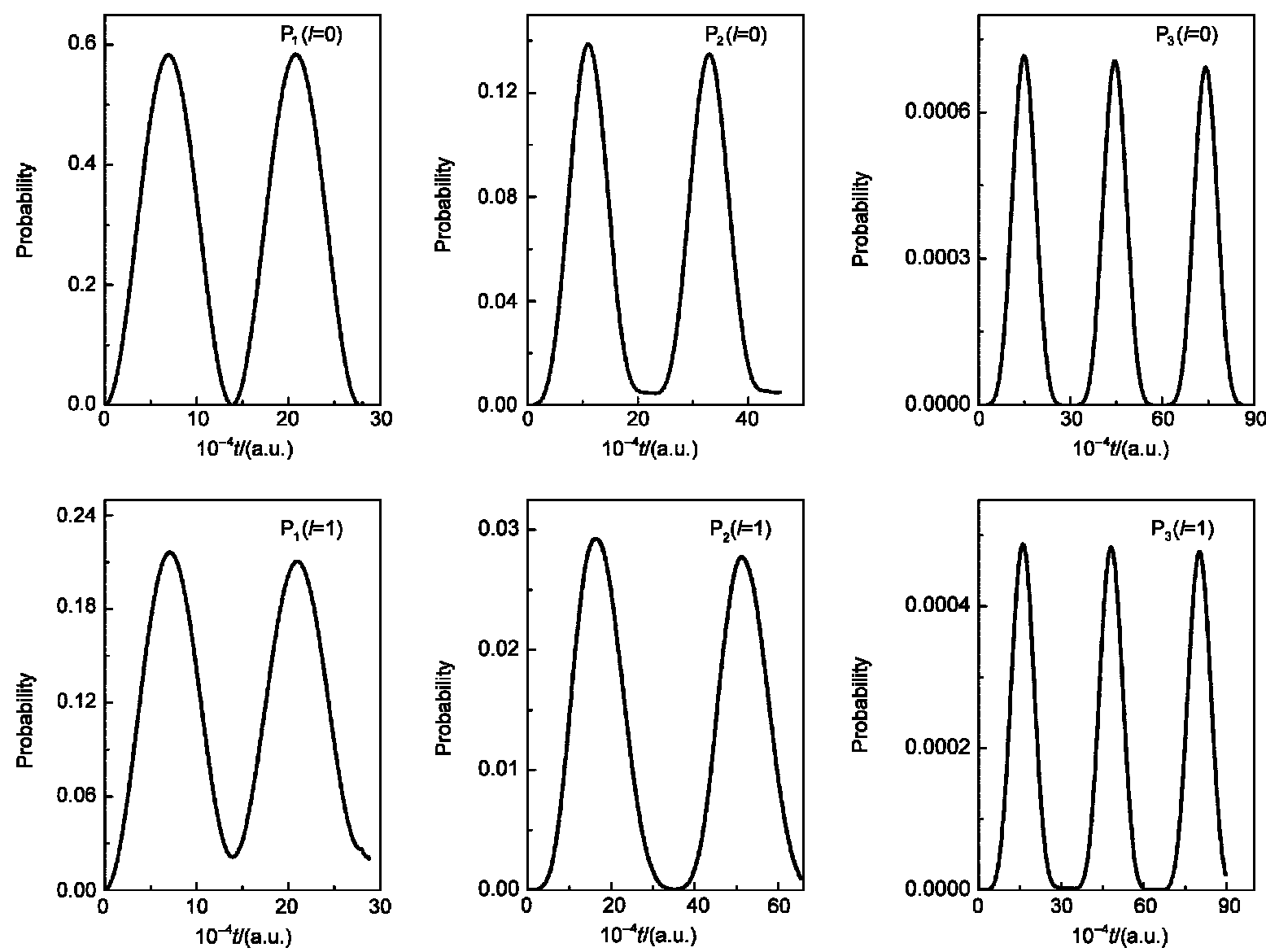

Fig.3 Average time-dependent transition probabilities for the one, two, and three-photon resonant transitions of OH as a function of time 

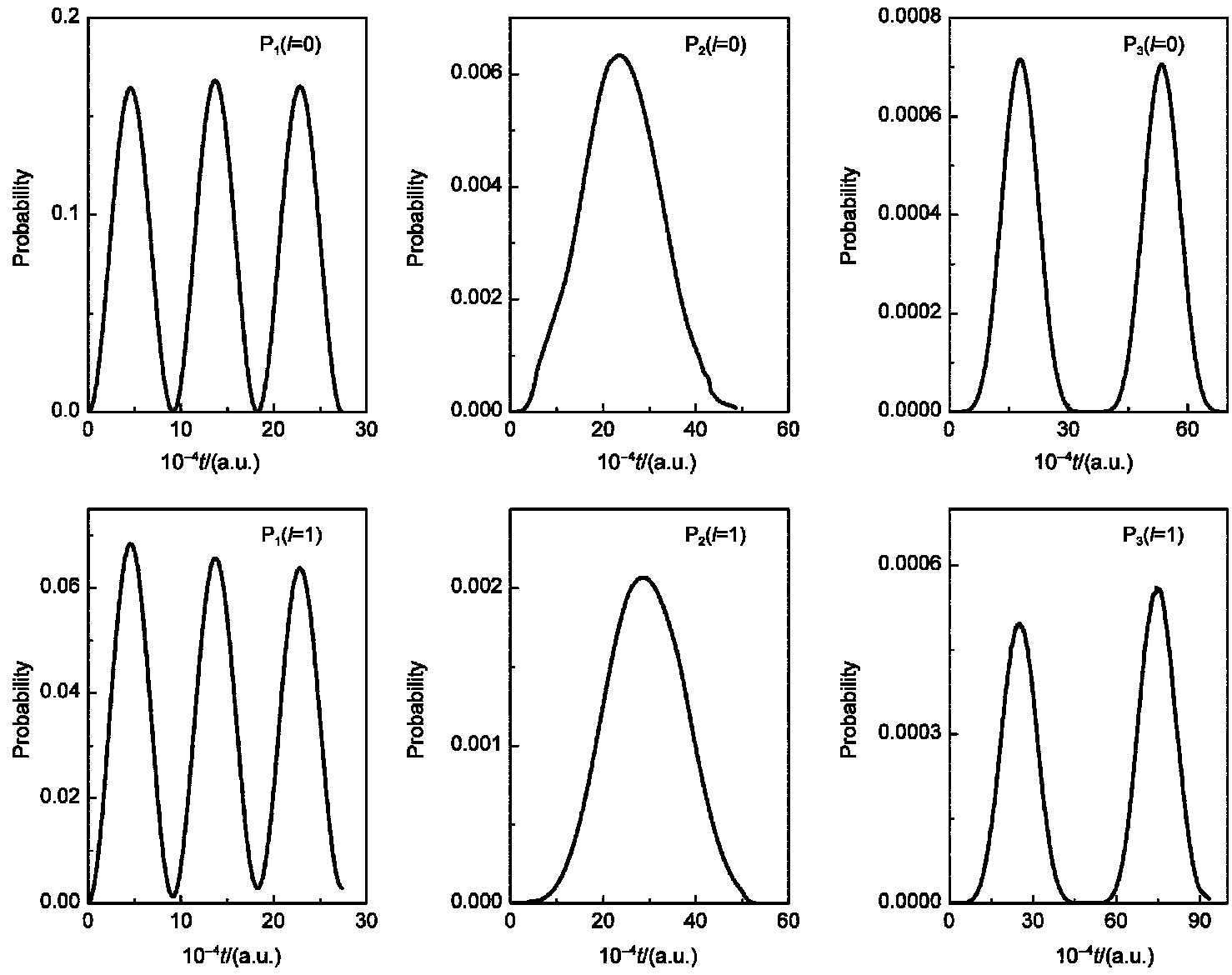

Fig.4 Average time-dependent transition probabilities for the one, two, and three-photon resonant transitions of OD as a function of time

is that although rotation energy has a little corrected action on molecular anharmonicity, the larger vibrational anharmonicity still cannot be overcome. Moreover, the anharmonicity of $\mathrm{OH}$ molecule is larger than that of OD molecule, so the influence of rotations on $\mathrm{OD}$ molecule is more obvious than that on $\mathrm{OH}$ molecule. This result is similar to a previously reported one. ${ }^{36}$

\subsection{Control of ro-vibrational multiphoton excitations}

In the previous section, we obtain the transition frequency of three-photon excitation and find the rotation energy has little corrected action on molecular anharmonicity. In order to observe correctional functions of the rotational energy and interference effect of molecular orientation on controlling multiphoton excitation, the three-photon excitations of $\mathrm{OH}$ and $\mathrm{OD}$ are taken as examples.

The laser field is

$$
E(t)=E_{0} s(t) \cos \Phi(t)
$$

where the laser shape functions are square-sinusoidal, Gaussian,

Table 1 Resonant transition periods (unit in a.u.) of $\mathrm{OH}$ and OD molecules

\begin{tabular}{lccccccc}
\hline & \multicolumn{3}{c}{$\mathrm{OH}$} & & \multicolumn{3}{c}{$\mathrm{OD}$} \\
\cline { 2 - 4 } \cline { 6 - 8 } \cline { 6 - 8 } & $\mathrm{P}_{1}$ & $\mathrm{P}_{2}$ & $\mathrm{P}_{3}$ & & $\mathrm{P}_{1}$ & $\mathrm{P}_{2}$ & $\mathrm{P}_{3}$ \\
\hline pure-vibration & $1.3 \times 10^{5}$ & $2.3 \times 10^{5}$ & $2.9 \times 10^{5}$ & & $1.3 \times 10^{5}$ & $3.3 \times 10^{5}$ & $3.3 \times 10^{5}$ \\
ro-vibration & $1.4 \times 10^{5}$ & $3.1 \times 10^{5}$ & $3.2 \times 10^{5}$ & & $1.4 \times 10^{5}$ & $5.1 \times 10^{5}$ & $5.0 \times 10^{5}$ \\
\hline
\end{tabular}

or triangular shapes, respectively.

$$
\begin{aligned}
& s_{1}(t)=\exp \left[-(t-\tau / 2)^{2} /(\tau / 4)^{2}\right] \\
& s_{2}(t)=\sin ^{2}(\pi t / \tau) \\
& s_{3}(t)=1-|2 t / \tau-1|
\end{aligned}
$$

in which $\tau$ is the laser pulse duration. $\Phi(t)$ is the phase of the laser field pulse as follows:

$$
\Phi(t)=\Phi_{0}+\int_{0}^{t}\left[\Delta \omega_{1}+\Delta \omega_{2} \mathrm{e}^{-\left(t^{\prime} / \tau\right)^{2}}\right] \mathrm{d} t^{\prime}
$$

where $\Phi_{0}$ is the laser initial phase. We further calculate threephoton excitation probabilities in the purely vibrational and rovibrational cases $(l=1)$ for comparison. When the rotation of the molecule is considered, the relationship between the molecular orientation and the polarized direction of the laser field becomes important. The maximum transition probabilities can be given as functions of the time and molecular orientation angle $\theta$. We first calculate three-photon excitation probabilities at different laser shapes when the molecular orientation angle $\theta$ is equal to zero. The laser parameters $\Delta \omega_{1}$ are the resonant transition frequency, and the chirped term $\Delta \omega_{2}$ and the laser pulse duration $\tau$ can be adjusted to obtain the optimum selective transition.

Fig.5 shows that the best selective three-photon excitation can be achieved when the laser shape is the Gaussian function. Although the three-photon excitation probability can reach a 


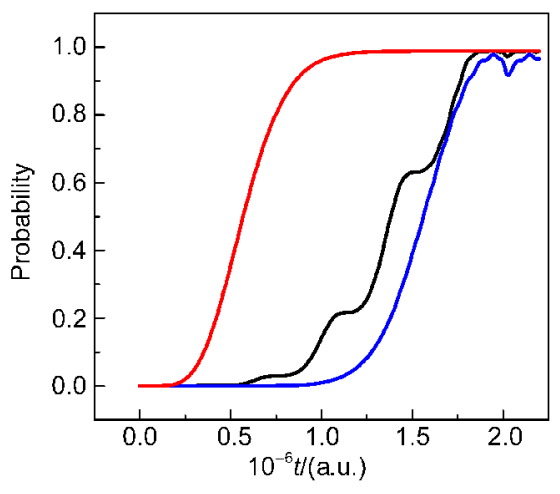

Fig.5 Maximum three-photon excitation probabilities as functions of the time by the three kinds of laser shaped and chirped pulses in $\mathrm{OH}$

The red line represents the Gaussian laser pulse shape, the blue line represents the triangular laser pulse shape, and the black line represents the squaresinusoidal laser pulse shape.

high value that is close to the one under the control of the square-sinusoidal and triangular laser pulse shape, oscillations of the population can be found in two cases. In addition, the three-photon excitation probability of the triangular shape case is smaller than those in the other two cases. Accordingly, we study the influence of rotations on controlling three-photon excitation under the Gaussian-shaped and chirped-laser pulse. We
Table 2 Optimum laser parameters (in a.u.) of $\mathrm{OH}$ and $\mathrm{OD}$ molecules

\begin{tabular}{ccccccc}
\hline \multirow{2}{*}{ The three-photon excitation } & \multicolumn{2}{c}{$\mathrm{OH}$} & & \multicolumn{2}{c}{ OD } \\
\cline { 2 - 3 } \cline { 5 - 6 } & $\Delta \omega_{1}$ & $\Delta \omega_{2}$ & & $\Delta \omega_{1}$ & $\Delta \omega_{2}$ \\
\hline pure-vibration & 0.0147070 & 0.0000038 & & 0.0111970 & 0.0000025 \\
ro-vibration & 0.0147069 & 0.0000033 & & 0.0111967 & 0.0000018 \\
\hline
\end{tabular}

can obtain complete three-photon vibrational excitation whether in the purely vibrational or in the ro-vibrational cases. The optimum laser parameters are given in Table 2; the pulse duration is $\tau=18 \times 10^{5}$ a.u., and the laser intensity is $I_{\max }=4.25 \times 10^{9} \mathrm{~W} \cdot$ $\mathrm{cm}^{-2}$ for $\mathrm{OH}$ and $I_{\max }=5.05 \times 10^{9} \mathrm{~W} \cdot \mathrm{cm}^{-2}$ for OD, respectively. Figs.6-9 show the maximum three-photon transition probabilities as functions of the time and molecular orientation angle $\theta$ in both $\mathrm{OH}$ and OD cases. We can also see that higher transition probabilities are achieved when the rotation is considered.

However, oscillations appear in the population which can be seen in Fig.7 and Fig.9, suggesting that rotational interference can decrease the selectivity of molecular multiphoton vibrational excitation. In addition, the highest excitation probability occurs only when the molecule is oriented along the direction of laser polarization. Fig.10 and Fig.11 show the initial laser phase dependence of the two cases, the maximum probabilities occur at different initial laser phases, which exhibit different modulation functions. In the interaction between the ultra-short
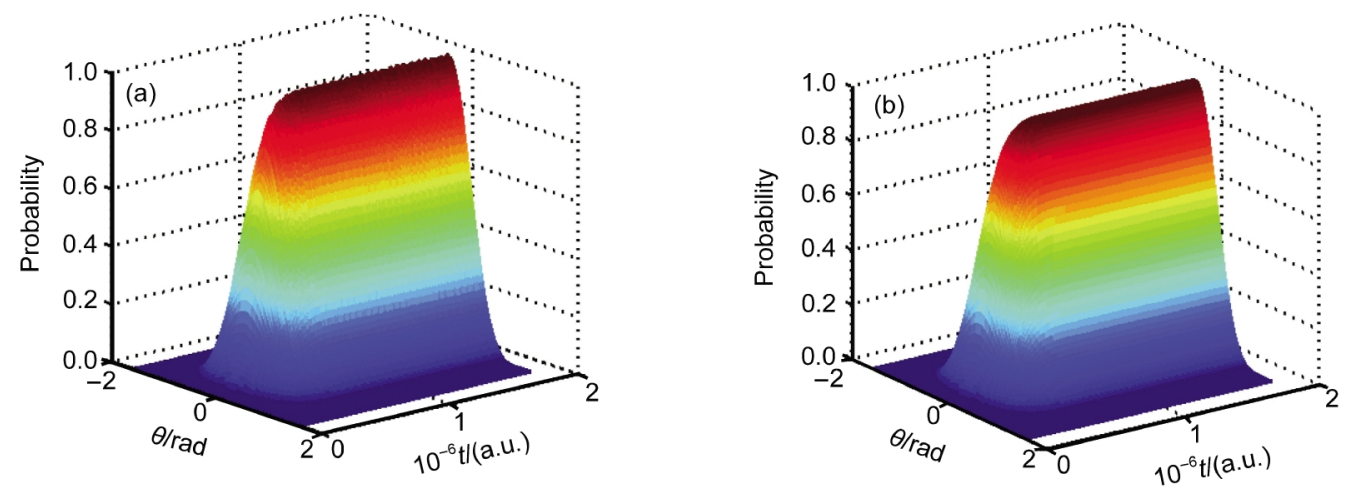

Fig.6 Maximum three-photon transition probabilities as functions of the time and molecular orientation angle $\theta$ by the Gaussian shaped and chirped pulse in $\mathrm{OH}$

(a) ro-vibrational case; (b) pure-vibrational case
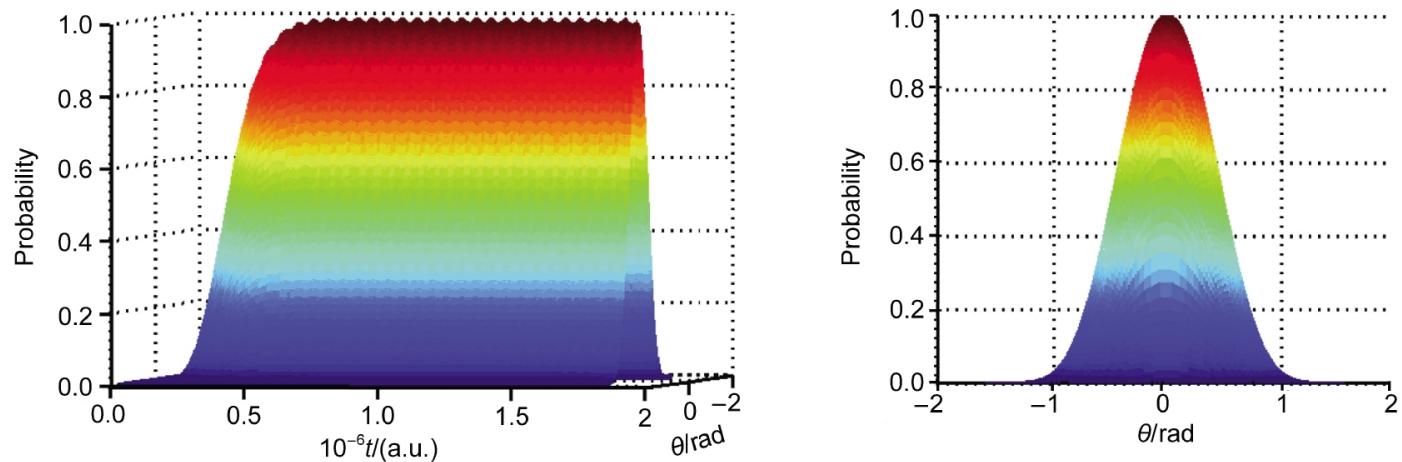

Fig.7 Control of three-photon vibrational transition with the molecular orientation angle using the Gaussian shaped and chirped pulse in the ro-vibrational case for $\mathrm{OH}$ 

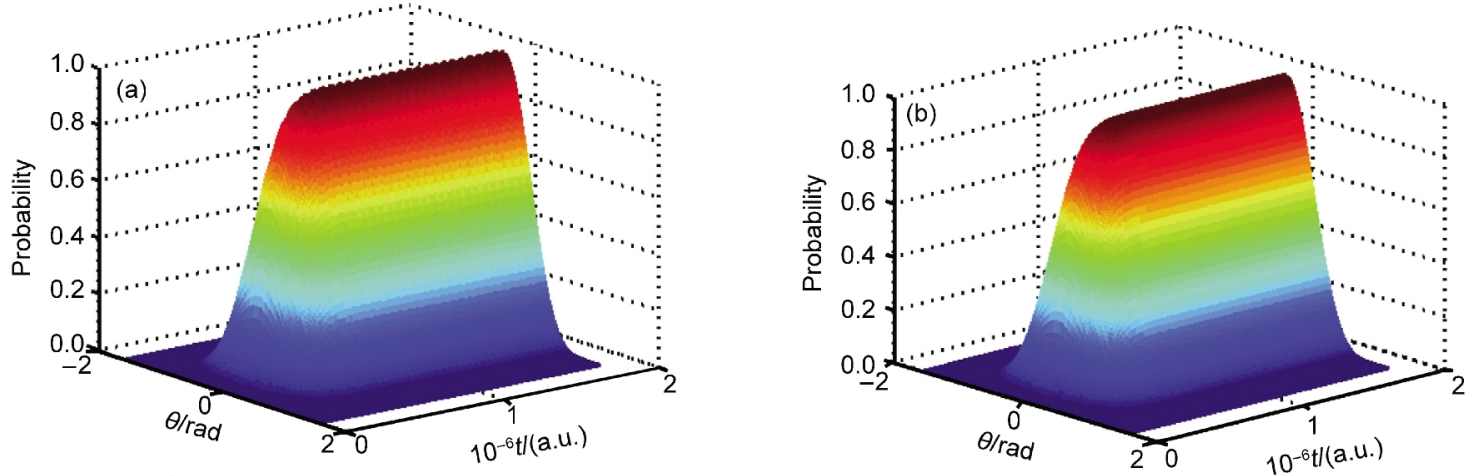

Fig.8 Maximum three-photon transition probabilities as functions of the time and molecular orientation angle $\theta$ by the Gaussian shaped and chirped pulse in OD

(a) ro-vibrational case; (b) pure-vibrational case
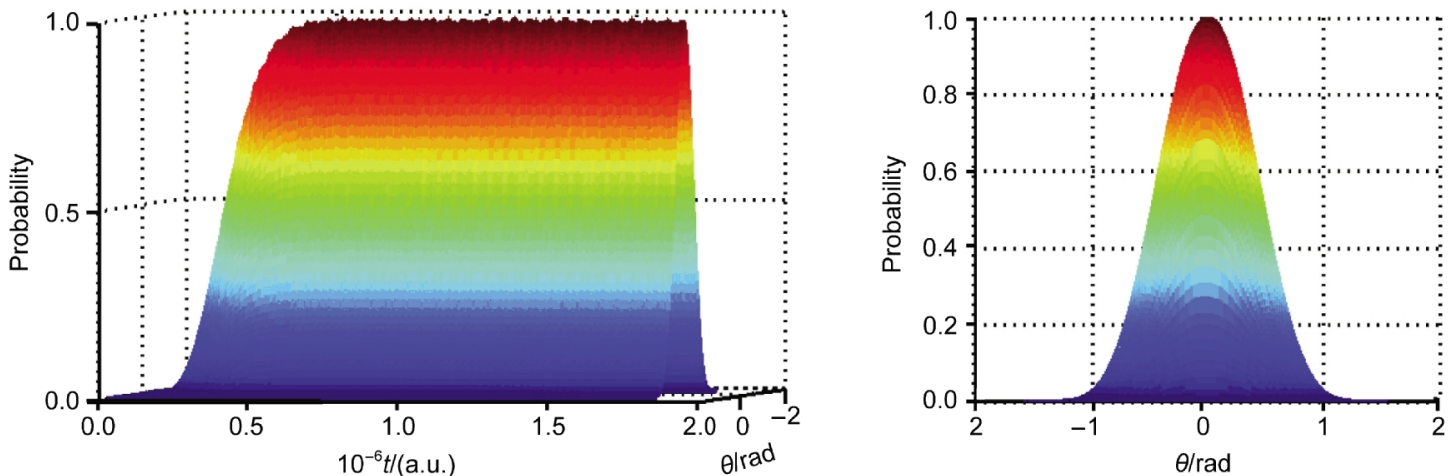

Fig.9 Control of three-photon vibrational transition with the molecular orientation angle using the Gaussian shaped and chirped pulse in the ro-vibrational case for $O D$

pulse and molecules, the frequency chirp is induced by the changes of laser initial phase, ${ }^{37-40}$ which produces modulation actions on the maximum excitation probabilities. A comparison of Fig.10(a) and Fig.10(b) indicates that the modulation actions in the ro-vibrational case are stronger than that in the pure-vibrational case. The range in the values of the maximum probabilities is from 0.96 to 1 in the ro-vibrational case, while the maximum probabilities range from 0.975 to 0.978 in the pure-vibrational case. The same result can be seen from the Fig.11(a, b). Furthermore, oscillations are found in Fig.10(a) and Fig.11(a), which reflect the sensitivity of the initial phase modulations in the non-resonant excitation. However, oscillations become stronger when the initial laser phase is at $\pi / 2$ or $3 \pi / 2$, and the oscillations in OD are smaller than those in $\mathrm{OH}$. This is an interesting phenomenon which is valuable to be further studied. We think that we should firstly confirm whether
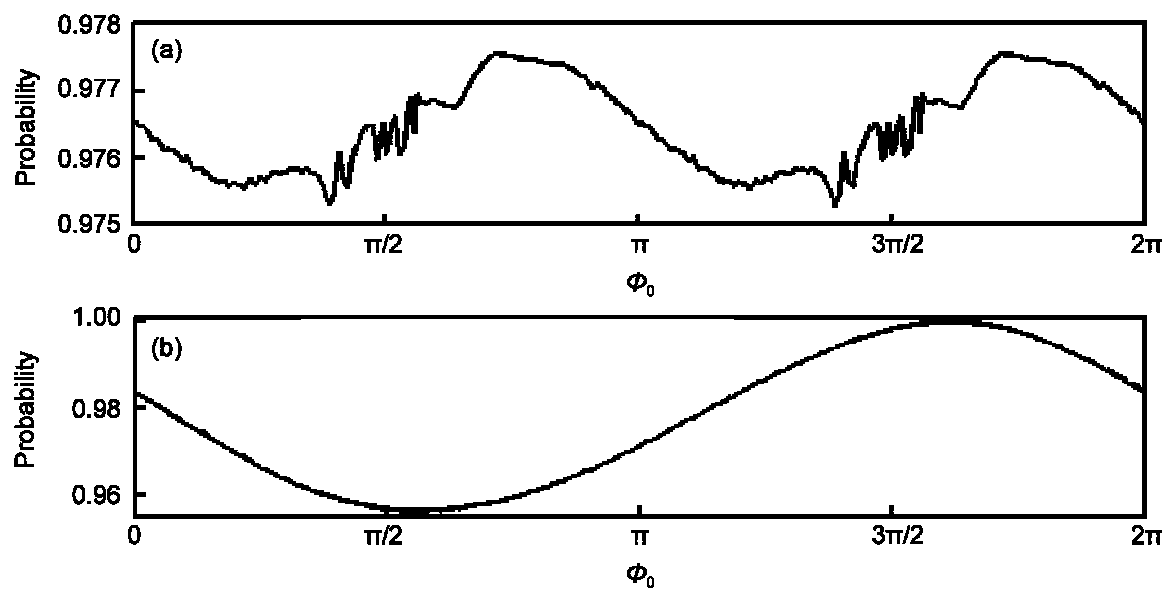

Fig.10 Initial laser phase dependence at the maximum of the selective three-photon vibrational transition probability of OH

(a) pure-vibrational case; (b) ro-vibrational case 

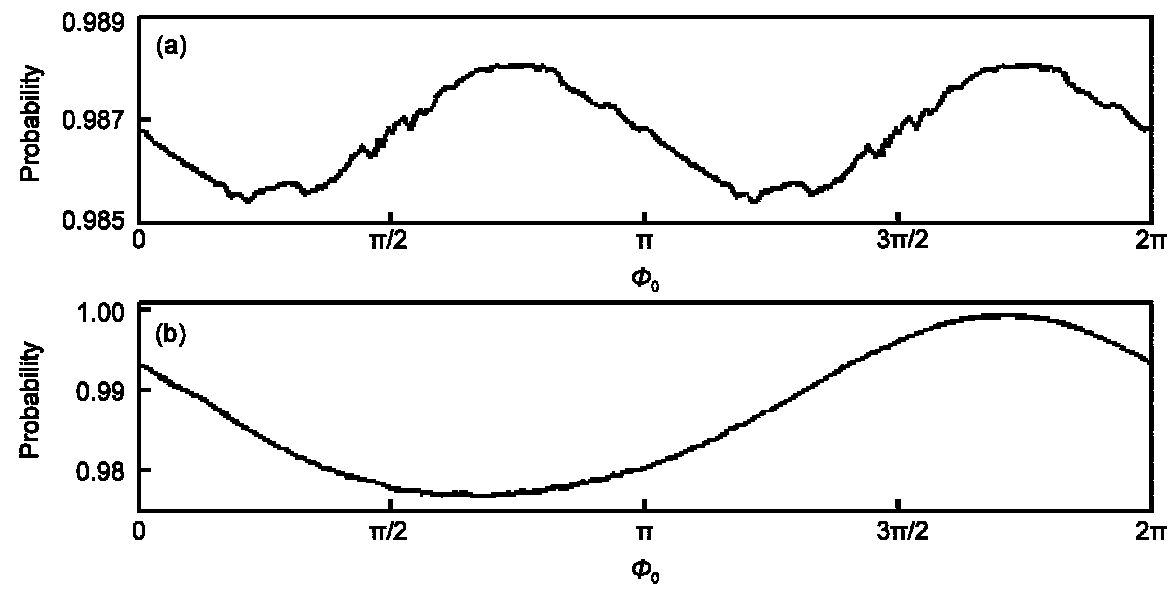

Fig.11 Initial laser phase dependence at the maximum of the selective three-photon vibrational transition probability of OD

(a) pure-vibrational case; (b) ro-vibrational case

the phenomenon happens in the others or more molecules and hope that the further explanations are given in the subsequent works.

\section{Conclusions}

We analytically study the influence of rotations and laser shapes on controlling infrared multiphoton processes in diatomic molecules using the Lie-algebraic approach. Results indicate that the molecular orientation in the laser field has a greater effect on transition probability, and that the effect of rotations on infrared multiphoton excitation depends on the anharmonicity of molecules. More importantly, control of molecular alignment and orientation is necessary to obtain multiphoton selective vibrational transition because the maximum value of multiphoton transition probability occurs only when the molecule is oriented along the direction of laser polarization. Furthermore, the rotational interference may decrease the selectivity of the molecular vibrational transition. However, the correct laser shape and initial laser phase may help achieve a better multiphoton vibrational transition on demand. This approach can be extended to triatomic molecules, and bending vibration can be considered.

\section{References}

(1) Kumarappan, V.; Holmegaard, L.; Martiny, C.; Madsen, C. B.; Kjeldsen, T. K.; Viftrup, S. S.; Madsen, L. B.; Stapelfeldt, H. Phys. Rev. Lett. 2008, 100, 093006. doi: 10.1103/ PhysRevLett.100.093006

(2) Chu, X. Phys. Rev. A 2008, 78, 043408. doi: 10.1103/ PhysRevA.78.043408

(3) Dimitrious, K. I.; Constantoudis, V.; Komninos, T.; Komninos, Y.; Nicolaides, C. A. Phys. Rev. A 2007, 76, 033406. doi: 10.1103/PhysRevA.76.033406

(4) Ramakrishna, S.; Seideman, T. Phys. Rev. Lett. 2007, 99, 113901. doi: 10.1103/PhysRevLett.99.113901

(5) Nakajima, K.; Abe, H.; Ohtsuki, Y. J. Phy. Chem. A 2012, 116 ,
11219. doi: 10.1021/jp3052054

(6) Kharin, V. Y.; Popov, A. M.; Ikhonova, T. V. Laser Physics 2012, 22, 1693. doi: 10.1134/S1054660X12110060

(7) Chu, X.; Groenenboom, G. C. Phys. Rev. A 2013, 87, 013434. doi: 10.1103/PhysRevA.87.013434

(8) Liu, B. K.; Wang, Y. Q.; Wang, L. Acta Phys. -Chim. Sin. 2010, 26, 3157. [刘本康, 王艳秋, 王 利. 物理化学学报, 2010, 26, 3157.] doi: 10.3866/PKU.WHXB20101220

(9) Iachello, F. Chem. Phys. Lett. 1981, 78, 581. doi: 10.1016/00092614(81)85262-1

(10) Van Roosmalen, O. S.; Benjamin, I.; Levine, R. D. J. Chem. Phys. 1984, 81, 5986. doi: 10.1063/1.447600

(11) Benjamin, I.; Levine, R. D.; Kinsey, J. L. J. Phys. Chem. 1983, 87, 727. doi: 10.1021/j100228a005

(12) Zheng, Y. J.; Ding, S. L. J. Chem. Phys. 1999, 111, 4466. doi: $10.1063 / 1.479210$

(13) Zheng, Y. J.; Ding, S. L. Phys. Rev. A 2001, 64, 032720. doi: 10.1103/PhysRevA.64.032720

(14) Zheng, Y. J.; Ding, S. L. Phys. Lett. A 1999, 256, 197. doi: 10.1016/S0375-9601(99)00207-8

(15) Qu, S. S.; Sun, W. G.; Wang, Y. J.; Fan, Q. C. Acta Phys. -Chim. Sin. 2009, 25, 13. [渠双双, 孙卫国, 王宇杰, 樊群超. 物理化 学学报, 2009, 25, 13.] doi: 10.3866/PKU.WHXB20090103

(16) Feng, H. R.; Ding, S. L. J. Phys. B 2007, 40, 69. doi: 10.1088/ 0953-4075/40/1/007

(17) Feng, H. R.; Liu, Y.; Zheng, Y. J.; Ding, S. L.; Ren, W. Y. Phys. Rev. A 2007, 75, 063417. doi: 10.1103/PhysRevA.75.063417

(18) Feng, H. R.; Cheng, J.; Yue, X. F.; Zheng, Y. J.; Ding, S. L. Chin. Phys. Lett. 2011, 28, 073301. doi: 10.1088/0256-307X/28/ 7/073301

(19) Levine, R. D. Chem. Phys. Lett. 1983, 95, 87. doi: $10.1016 /$ 0009-2614(83)85071-4

(20) Levine, R. D. Intramolecular Dynamic, 1st ed.; Reidel: Dordrecht, 1982.

(21) Cooper, I. L.; Gupta, R. K. Phys. Rev. A 1997, 55, 4112 
doi: 10.1103/PhysRevA.55.4112

(22) Cooper, I. L. J. Phys. Chem. A 1998, 102, 9565. doi: 10.1021/ jp982149r

(23) Rau, A. R. P.; Zhao, W. C. Phys. Rev. A 2005, 71, 063822. doi: 10.1103/PhysRevA.71.063822

(24) Alhassid, Y.; Levine, R. D. Phys. Rev. A 1978, 18, 89. doi: 10.1103/PhysRevA.18.89

(25) Wei, J.; Norman, E. Proc. Am. Math. Soc. 1964, 15, 327. doi: 10.1090/S0002-9939-1964-0160009-0

(26) Korolkov, M. V.; Paramonov, G. K. Phys. Rev. A 1997, 56, 3860. doi: 10.1103/PhysRevA.56.3860

(27) Stranges, S.; Rithcer, R.; Alagia, M. J. Chem. Phys. 2002, 116 , 3676. doi: $10.1063 / 1.1448283$

(28) Amstrup, B.; Henriksen, N. E. J. Chem. Phys. 1992, 97, 8285. doi: $10.1063 / 1.463399$

(29) Elghobashi, N.; Krause, P.; Manz, J.; Oppel, M. Phys. Chem. Chem. Phys. 2003, 5, 4806. doi: 10.1039/b305305a

(30) Herzberg, G. Molecular Spectra and Molecular Structure I. Spectra of Diatomic Molecules, 1st ed.; D. Van Mostrand Company, Inc.: Princeton, 1950; pp 560, 106.

(31) Jakubetz, W.; Just, B.; Manz, J.; Schreier, H. J. J. Phys. Chem.
1990, 94, 2294. doi: 10.1021/j100369a019

(32) Dai, Y.; Ding, S. L. Int. J. Quantum Chem. 1999, 71, 201. doi: 10.1002/(SICI)1097-461X(1999)71:2<201::AID-QUA9> 3.0.CO;2-A

(33) Broeckhove, J.; Feyen, B.; Van Leuven, P. Int. J. Quantum Chem. 1994, 52, 173. doi: 10.1002/qua.560520818

(34) Geng, Z. H.; Dai, Y.; Ding, S. L. Chem. Phys. 2002, 278, 119 doi: 10.1016/S0301-0104(02)00404-4

(35) Walker, R. B.; Preston, R. K. J. Chem. Phys. 1977, 67, 2017. doi: $10.1063 / 1.435085$

(36) Chang, J.; Wyatt, R. E. J. Chem. Phys. 1986, 85, 1840. doi: $10.1063 / 1.451185$

(37) Bartels, R. A.; Weinacht, T. C.; Wagner, N.; Baertschy, M.; Greene, C. H.; Murnane, M. M.; Kapteyn, H. C. Phys. Rev. Lett. 2001, 88, 013903. doi: 10.1103/PhysRevLett.88.013903

(38) Comstock, M.; Lozovoy, V. V.; Dantus, M. Chem. Phys. Lett. 2003, 372, 739. doi: 10.1016/S0009-2614(03)00489-5

(39) Diels, J. C.; Rudolph, W. Ultrashort Laser Pulse Phenomena, 2nd ed.; Academic Press: Burlington, 2006; pp 44-46.

(40) Boyd, R. W. Nonlinear Optics, 3rd ed.; Academic Press: Burlington, 2008; pp 69-122. 